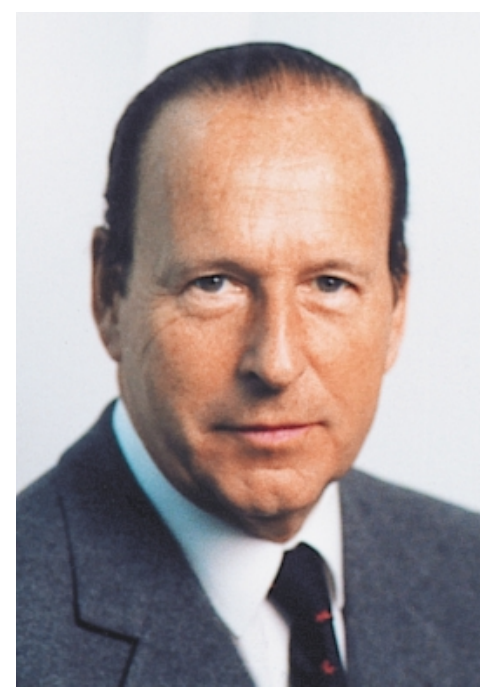

\title{
Bilaterale Abkommen: Ein schlechtes Vertrags- werk auch für die Ärzte
}

\author{
J. H. Dunant
}

Korespondenz:

PD Dr. med. Jean Henri Dunant

Nationalrat SVP

Luftmattstrasse 12

CH-4052 Basel

Grundsätzlich wären Verträge mit der EU ein erwünschter Schritt zur Verbesserung und zum Ausbau der gegenseitigen Beziehungen. Nachfolgend möchte ich kurz ausführen, weshalb es mir wichtig erscheint, dass das Volk über derart schwerwiegende Veränderungen abstimmen kann. So führt der Transitverkehr $\mathrm{zu}$ erheblichen Problemen mit einer Lastwagenflut und dadurch bedingten finanziellen Lasten. Die Schweiz wird zukünftig jährlich rund 300 Millionen Franken als flankierende Massnahmen ausgeben, um den Verkehr vermehrt von der Strasse auf die Schiene zu bringen. Die 40-Tonnen-Lastwagenflut wird an den bestehenden Brücken- und Strassenkonstruktionen schwere Schäden verursachen, was wiederum erhebliche Mehrkosten nach sich zieht, von der Luftverschmutzung nicht zu sprechen. Es ist eine Illusion zu glauben, dass der Verkehr im erwarteten Ausmass auf die Schiene gebracht werden kann. Die freie Konkurrenz in der Landwirtschaft bringt zwar dem Konsumenten tiefere Lebensmittelpreise, verursacht jedoch Probleme und Kosten im Sektor Landwirtschaft zu Ungunsten der Bauern. Von den bilateralen Verträgen profitiert die grosse Wirtschaft. KMU, Mittelstand und Selbständigerwerbende haben das Nachsehen.

Unmittelbar betroffen sind die Ärzte durch den freien Personenverkehr. Die Vorstellung, dass man nach sieben Jahren durch ein fakultatives Referendum eine Notbremse anziehen könne, ist naiv, würden doch durch ein Nein alle anderen sechs Verträge ausser Kraft gesetzt. Ab dem Jahr 2001 werden Diplome ge- genseitig anerkannt. Dies würde dazu führen, dass unser hochqualifizierter Ärztestand unterlaufen wird und dass ein Nivellierungseffekt stattfindet. In den nächsten Jahren ist mit einem Zuwachs von ca. 380 praktizierenden Schweizer Ärzten pro Jahr zu rechnen. Ab 2001 kommen zwischen 30 und 215 Praxiseröffnungen durch bereits in der Schweiz arbeitende ausländische Ärzte pro Jahr hinzu. Nach ca. 2007 werden Euro-Docs ebenfalls eine Praxis in der Schweiz eröffnen können. Hier wird sich die Zahl in Grenzen halten, da diese Ärzte ausbildungsmässig nicht auf Schweizer Niveau FMH sein werden. Die durch den Ärztezuwachs bedingten Kosten im Gesundheitswesen werden sich bei konstanter Entwicklung auf ca. 265 Millionen Franken pro Jahr, also 1 Milliarde alle vier Jahre, belaufen. Die Gesamtzahl praktizierender Ärzte nimmt jährlich konstant um bisher 300 bis 400 zu, zukünftig um 500 bis 600. Der Anreiz, in der Schweiz eine neue Existenz aufzubauen, ist gross, zumal das soziale Netz in unserem Land grosse Vorteile bietet. Der freie Personenverkehr wird zu einer Überflutung unserer Universitäten führen. Gegenwärtig befinden sich 15000 Studenten/-innen aus der EU in der Schweiz. Ein Numerus clausus wird nicht zu verhindern sein, und der Ausbau der Universitäten führt zu weiteren finanziellen Belastungen.

Das Gesamtpaket der sieben Verträge ist nachteilig für die Schweiz. Nur ein klares Nein bei der Abstimmung vom 21. Mai 2000 kann einen EU-Beitritt für die nächsten Jahre verhindern. 